East African Medical Journal Vol. 81 No. 2 February 2004

CARDIOVASCULAR RISK FACTOR PROFILE OF BLACK AFRICANS UNDERGOING CORONARY ANGIOGRAPHY

C. Kamotho, BSc., MBChB, DTM\&H, Registrar, Kenyatta National Hospital, P.O Box 55650, Nairobi, Kenya, E. O. Ogola, MBChB, MMed, Chairman, Department of Medicine, M. Joshi, MBChB, MMed, MPH (Epi), Lecturer, Department of Medicine, College of Health Sciences, University of Nairobi, P.O. Box 19676, Nairobi, Kenya and D. Gikonyo, MBChB, MMed, Consultant Cardiologist, The Nairobi Hospital, P.O. Box 30026, Nairobi, Kenya

Request for reprints to: Dr. C. Kamotho, P.O. Box 55650, Nairobi, Kenya

\title{
CARDIOVASCULAR RISK FACTOR PROFILE OF BLACK AFRICANS UNDERGOING CORONARY ANGIOGRAPHY
}

\author{
C. KAMOTHO, E. O. OGOLA, M. JOSHI and D. GIKONYO
}

\begin{abstract}
Background: Coronary artery disease (CAD) is a growing epidemic on the African continent. It remains uncertain whether the risk factors identified as contributing to CAD in white populations contribute to a similar extent to CAD incidence in black populations. No data of the local population exists that is based on the coronary angiogram (CA).

Objectives: To analyse the relationship of conventional cardiovascular risk factors with presence of CAD in black Africans.

Design: This was a dual-armed study, consisting of retrospective and prospective comparative arms.

Subjects: Black Africans who underwent coronary angiography.

Setting: Nairobi Hospital, Cathereterization laboratory.

Main outcome measures: The conventional risk factors: age, male gender, hypertension, obesity, smoking, diabetes mellitus, dyslipidaemia, alcohol use and interventricular septum (IVS) hypertrophy, as a marker of LVH.

Results: One hundred and sixty nine patients fulfilled the inclusion criteria; 144 in the retrospective arm and 25 in the prospective. The larger retrospective arm showed that the group with CAD, compared to the normal group, was significantly older, with a higher mean age of 54.4 years compared to 49.8 years $(P=0.005)$; had significantly more males, with a male to female ratio of 5.5:1 compared to $2.3: 1 \quad(P=0.045)$; had a very significantly larger proportion of diabetics $(38.5 \%$ compared to $12 \%, P=0.0002)$, and also had a significantly larger proportion of patients with dyslipidaemia $(67.3 \%$ compared to $35.9 \%, P=0.0003$ ). The percentage of hypertensives was high in both groups, with $(65.4 \%)$ in the CAD group and $62 \%$ in the Normal group being hypertensive $(\mathbf{P}=\mathbf{0 . 6 8})$. The percentage of smokers was small in both groups, being $15.4 \%$ and $13 \%$ respectively. Smoking, increased BMI, alcohol use, and increased IVS were each found to be distributed equally in both groups. In addition, the Waist hip ratio (WHR) and waist circumference (WC) did not differ significantly between the two groups studied. Conclusions: The risk factors found to be most strongly associated with presence of angiographically-detected CAD in the population studied were diabetes mellitus, dyslipidaemia, age and male gender. There was a high prevalence of hypertension, with equal distribution in both groups under study; hence this risk factor was not discriminatory for CAD. There was a low prevalence of cigarette smoking in this particular study; it was not predictive of presence of CAD.
\end{abstract}

\section{INTRODUCTION}

Coronary artery disease is a growing epidemic on the African continent. Indeed, over the last thirty years morbidity and mortality due to cardiovascular disease have increased rapidly in developing countries(1). The risk factors that have been shown to influence the development of CAD in white populations are hypertension, hypercholesterolaemia, low levels of highdensity lipoprotein (HDL) cholesterol, cigarette smoking, diabetes mellitus, age and male gender. Moreover, the large variation in the average extent of coronary atherosclerosis among black populations, as well as among white populations, suggests that interplay between genetic factors and environmental factors might determine the severity and extent of atherosclerotic lesions within both groups(2). It remains uncertain whether the risk factors identified as contributing to CAD in white populations contribute to a similar extent to CAD incidence in black populations(3). No data of the local population exists that is based on the Coronary Angiogram (CA), the current "Gold Standard" diagnostic test for coronary artery disease. This study sought to identify those cardiovascular risk factors that are 
particularly associated with the presence of coronary artery disease (CAD) in black Africans in Kenya. It was based on the coronary angiogram (CA).

The objective of the study was to analyse the relationship of conventional cardiovascular risk factors with presence of CAD in black Africans. Specifically: i) To describe the prevalence of conventional cardiovascular risk factors in black Africans with CAD as documented on coronary angiography at the Nairobi Hospital Catheterization laboratory.

ii) To describe the prevalence of the same risk factors in Black Africans with normal coronary arteries as documented on CA at the Catheterization laboratory. iii) To compare the prevalence of the said risk factors between the two groups.

\section{MATERIALS AND METHODS}

This was a comparative study consisting of a retrospective and a prospective arm. In the retrospective arm all those patients who had undergone a CA at the Nairobi Hospital Cardiac Catheterization Laboratory between its inception in April 1996 to December 2001 were included who fulfilled the inclusion criteria, viz to be of Black race, and consent by the cardiologist to include the data from the files.

Similarly, for the prospective arm, all those patients who underwent a CA during a six-month study period between October 2002 and March 2003, and gave informed consent, were included. Every morning the list of names of patients who had been booked for a coronary angiogram were obtained from the Catheterization laboratory of the Nairobi Hospital, together with the name of the referring cardiologist or clinic. The patients were interviewed before or after the procedure, either at their respective clinics or at the Nairobi Hospital, and were invited to take part in the study. After informed consent, an investigator administered-questionnaire was filled in. The principal investigator participated in the angiograms, and carried out other modes of cardiovascular evaluation of the patients, such as echocardiography.

The risk factors analysed were: age, male gender, hypertension, obesity, smoking, diabetes mellitus, dyslipidaemia, alcohol use and IVS hypertrophy, as a marker of LVH. Data was retrieved from the case files, and diagnoses such as hypertension, dyslipidaemia, were obtained from case notes. JNC VI criteria were followed for a diagnosis of hypertension: Systolic Blood Pressure (SBP) $>/=140 \mathrm{mmHg}$ and Diastolic Blood Pressure (DBP) $>/=90 \mathrm{mmHg}$, or the current use of anti-hypertensive therapy(4). Dyslipidaemia was diagnosed according to the second report of the National Cholesterol Education Programme(5). For the Coronary Angiogram, abnormal was taken as $>/=50 \%$ stenosis on one or more of the coronary arteries, judged by the standard method for interpreting the presence and severity of stenoses in the epicardial coronary arteries, which continues to be visual assessment or "eyeballing." Those arteries with less than $50 \%$ are defined as sub-critical(6).

Ethical approval: Ethical approval was obtained from the Nairobi Hospital Education Committee.

Data analysis: Continuous data were analysed into means and categorical data into percentages, with the corresponding $95 \%$ confidence intervals. Comparisons of continuous data were made using the Student t-test, and those of categorical data using the Chi-square test or Fischer's exact test. Correlations between variables were tested using the Pearson correlation coefficient. Prevalence rates of risk factors were calculated as percentages with 95\% confidence intervals. Statistical significance was defined as a two-tailed p-value of less than or equal to 0.05 .

\section{RESULTS}

For the retrospective arm, a total of 662 angiograms were carried out between April 1996 and December 2001(7). Four hundred and fifty two (68\%) were nonBlack. Of the 210 black patients, consent to review the files of patients was given by six out of the seven consultants involved. Finally, therefore, 144 patients fulfilled the inclusion criteria and were included in the retrospective arm of the study. Of these, 52(36.1\%) had abnormal angiograms, $86(59.7 \%)$ had normal studies, and six $(4.2 \%)$ had sub-critical lesions.

Meanwhile, during the six-month prospective study period between October 2002 and March 2003, 62 patients underwent coronary angiograms at the Nairobi Hospital Catheterization laboratory. Twenty nine (47\%) were black, and four did not give consent. Data were thus eventually collected prospectively for 25 patients. Of these, $18(72 \%)$ had CAD, three (12\%) were normal, and four had sub-critical stenoses.

The larger retrospective arm, on which the following results are based, showed (Table 1) that the group with CAD, compared to the Normal group, had a very significantly larger proportion of diabetics $(38 \%$ in the CAD group compared to $12 \%, \mathrm{P}=0.0002$ ) and patients with dyslipidaemia $(67.3 \%$ compared to $35.9 \%$, $\mathrm{P}=0.0003$ ); the group was significantly older (mean age 54.4 years compared to $49.8, \mathrm{P}=0.005$ ); and had a significantly higher proportion of males (M:F ratio of 5.5:1 compared to $2.3: 1, \mathrm{P}=0.045)$. The percentage of hypertensives was high in both groups: $65.4 \%$ in the CAD group and $62 \%$ in the Normal group $(\mathrm{P}=0.68)$. The percentage of smokers was small in both groups, being $15.4 \%$ and $13 \%$ respectively. Both presence of hypertension and smoking were not discriminative for CAD. Neither was there a difference in prevalence of obesity (assessed by BMI, WHR and WC), alcohol use and increased IVS in each group. The presenting complaints and clinical events that prompted an investigative pathway culminating in a coronary angiogram study on the patients are summarised in Table 2 . 
Table 1

Summary of results in retrospective arm

\begin{tabular}{|c|c|c|c|}
\hline \multirow[b]{2}{*}{ Variable } & \multicolumn{2}{|c|}{ Coronary Angiogram } & \multirow[b]{2}{*}{ P-value } \\
\hline & $\begin{array}{l}\text { CAD } \\
\mathrm{n}=52(36.1 \%)\end{array}$ & $\begin{array}{l}\text { Normal } \\
\mathrm{n}=92(63.9 \%)\end{array}$ & \\
\hline Prevalence of diabetics & 38.5 & 12.0 & 0.0002 \\
\hline $\begin{array}{l}\text { Prevalence of patients with } \\
\text { dyslipidaemia }\end{array}$ & 67.3 & 35.9 & 0.0003 \\
\hline Mean Age (years) & 54.4 & 49.8 & 0.005 \\
\hline Male to female ratio & $5.5: 1$ & $2.3: 1$ & 0.045 \\
\hline Percentage of hypertensives & 65.4 & 62.0 & 0.68 \\
\hline Mean BMI $(n=125)$ & 26.55 & 27.97 & 0.259 \\
\hline Mean WHR (prospective) & 0.94 & 0.95 & 0.737 \\
\hline Mean WC $(\mathrm{cm})$ (prospective) & 107.3 & 101.3 & 0.143 \\
\hline \multicolumn{4}{|l|}{ Prevalence of smokers } \\
\hline Current & 15.4 & 13.0 & 0.227 \\
\hline Prevalence of alcohol users & 32.7 & 36.9 & 0.67 \\
\hline Mean IVS: $(\mathrm{cm})$ & 1.13 & 1.02 & 0.075 \\
\hline
\end{tabular}

Table 2

Indications for coronary angiogram in the retrospective arm

\begin{tabular}{llll}
\hline $\begin{array}{l}\text { Presenting } \\
\text { complaints }\end{array}$ & $\begin{array}{l}\text { Abnormal } \\
\text { CA } \% \text { of Abnormal })\end{array}$ & $\begin{array}{l}\text { Normal } \\
\text { CA }(\% \text { of Normal })\end{array}$ & Total \\
\hline $\begin{array}{l}\text { Stable Angina } \\
\text { Pectoris }\end{array}$ & $20(38.5 \%)$ & $12(13.0 \%)$ & $32(22.2 \%)$ \\
$\begin{array}{l}\text { Positive exercise } \\
\text { stress test }\end{array}$ & $10(19.2 \%)$ & $28(30.4 \%)$ & $38(26.4 \%)$ \\
$\begin{array}{l}\text { Atypical chest pains } \\
\text { Myocardial infarct }\end{array}$ & $9(17.3 \%)$ & $31(33.7 \%)$ & $40(27.8 \%)$ \\
$\begin{array}{l}\text { Pre-Op Evaluation } \\
\text { Unstable Angina Pectoris }\end{array}$ & $8(15.4 \%)$ & $5(5.4 \%)$ & $13(9.0 \%)$ \\
$16(17.4 \%)$ & $19(13.2 \%)$ \\
Total & $2(3.8 \%)$ & $(0 \%)$ & $2(1.4 \%)$ \\
\hline
\end{tabular}

\section{DISCUSSION}

The established cardiovascular risk factors have been determined from several well-conducted landmark studies. However, these have centered mainly on populations from Western Europe and North America. Sub-Sahara Africa is lacking in such studies, which are pertinent, given the differences in genetic heritage and lifestyle from the typical Western populace.

This study, the first of its kind in the region of East and Central Africa, was based on the CA, the current "Gold Standard" diagnostic test for CAD. It set out to describe the distribution of coronary risk factors in black patients with established CAD. It did not seek to explore or define the role of these risk factors in the pathogenesis of CAD, hence it cannot discount a factor as non-contributory to development of CAD.

Approximately one third of the patients who underwent a CA during the time period of the retrospective arm were black, the remainder were Asian and European. The proportion of blacks in the prospective arm was slightly higher. This could in part be explained by the increasing accessibility of such diagnostic means as $\mathrm{CA}$, and to a greater awareness among health-care workers of the rising prevalence of coronary artery disease among those of Black race.

A strong association was found between diabetes mellitus and coronary artery disease. A significantly higher proportion of those with CAD were diabetic as compared to those with normal coronary arteries.

These findings are in keeping with previous findings that insulin resistance, hyperinsulinaemia and glucose intolerance appear to promote atherosclerosis $(8,9)$. In the Framingham Heart Study, for example, diabetes, impaired glucose tolerance, and high normal levels of glycosylated haemoglobin were powerful contributors to atherosclerotic cardiovascular events, particularly in women $(10,11)$.

Dyslipidaemia was shown to be highly predictive of CAD, and this is consistent with Western data. A stratified analysis of the various types of dyslipidaemia could not, however, be drawn from this study, because 
most of the data were retrospective, and the prospective data were lacking in statistical power. Also, some of the patients were on lipid-lowering therapy.

Age was noted to be an important discriminatory factor in this study population. Indeed, the mean age of the patients who had coronary artery disease was rather low compared to the ages mentioned in Western data(12). Hence the importance of increased awareness of CAD even among the relatively young.

The proportion of males among those who underwent $\mathrm{CA}$ was three times that of females. This may be a reflection of the skew in exposure to cardiovascular risk factors that males have been subjected to. With an average age in the fifties, these could be among those first few well-educated men who got jobs in the civil service soon after independence, and adopted an urban Western lifestyle. Meanwhile, most women of the same ages tended to remain in the rural homes. With an increased presence of women in professional lives, an increase in coronary symptomatology and need for catheterization is expected in this group in the future.

While hypertension is a well-established coronary risk factor(13), there was no major difference noted in the prevalence of hypertension between the two groups under study. This study did, however, reveal a high prevalence of hypertension among all the patients who underwent a coronary angiogram. A local study revealed a high proportion of patients with hypertension and one or more other cardiovascular risk factors(14).

The results show that hypertension was not a discriminatory risk factor for the presence of coronary heart disease in the population studied. Although hypertension is a major risk factor per se for stroke and heart failure, it is a significant coronary risk factor especially so when associated with target organ damage(15). Probably the patients in question suffered little, if any, of this. Also, the high prevalence of hypertension among the patients would necessitate a larger study to show a difference between the two groups.

Obesity: The retrospective arm showed an unexpected trend in the BMI and WHR, with the normal CA groups in both arms having values that were (non-statistically) higher or equal to the values in the CAD group. The WC (prospective arm) on the other hand showed a positive correlation (albeit not statistically significant) with CAD.

The unexpected trend in the first two parameters (BMI and WHR) could be explained by the higher proportion of premenopausal women in the normal group than in the CAD group. The low average age includes women who still enjoy hormonal cardiovascular protection, and, as shown in a local study on diabetics, females were significantly more likely to have abnormal WHR's(16). Therefore this proportion increased the parameters in the normal group.
Cigarette smoking: This study manifests a rather low prevalence of smokers in the population studied. This is in keeping with a Nigerian study of cardiovascular risk factors in middle-aged Nigerians, which illustrated, among the 146 persons studied, a $0 \%$ prevalence of smokers of more than 10 sticks per day (17). Cultural and religious reasons could have a role in maintaining the low prevalence. However, with the increased marketing in Africa by tobacco companies, and the rising numbers of women smokers, the epidemiology of smoking and the pattern of its deleterious effects could change within the next few years.

Thus while this study did not reveal cigarette smoking to be a discriminatory factor for CAD, cigarette smoking remains an important and reversible risk factor for CAD. The thickness of the interventricular septum was assessed by echocardiography. Increased thickness served as a surrogate marker of left ventricular hypertrophy. The mean IVS values in the CAD group were slightly higher than those of the normal group, with a p-value tending towards significance.

One of the limitations of the study was selection bias. While the target population was the black African population in Kenya, the sample studied was a highly select group, namely the patients undergoing a CA at the Nairobi Hospital Catheterization Laboratory. These were obtained after a multi-step selection process, hence the potential for introduction of bias was high.

The results accruing from this study, however, save for those to do with obesity are consistent with established knowledge about risk factors that has been gathered from the study of large free-living populations. This suggests that this sample is closely representative of the target population.

\section{CONCLUSION}

(i). The risk factors most strongly associated with presence of coronary artery disease in this population were diabetes mellitus and dyslipidaemia. These two conditions are thus strongly predictive of CAD demonstrated at CA.

(ii). Age and male gender were found to be strongly associated with CAD.

(iii). There was a high prevalence of hypertension in the population studied. There was, however, equal distribution of this risk factor in the two groups, and thus it was not found to be discriminately associated with CAD in this particular study.

(iv). Tobacco use and BMI were not found to be particularly associated with CAD compared to the Normal group in this study. There was a low prevalence of cigarette use in this study.

\section{RECOMMENDATIONS}

(i). Primary and secondary prevention of coronary artery disease should be carried out vigorously in blacks with diabetes mellitus and / or dyslipidaemia. 
(ii). Despite not being found to be associated with CAD in this study, given its high overall prevalence, hypertension should be identified and treated to lower overall cardiovascular risk.

(iii). More prospective studies should be carried out with a wider sample base and larger numbers, to further elucidate the significance of particular risk factors, such as smoking.

(iv). Lifestyle changes and education should be encouraged to attain and maintain recommended habits and anthropometric parameters. The opportunity exists in our population to combat, for example, the expected rise in cigarette smoking prevalence along with its attendant risks.

\section{ACKNOWLEDGEMENTS}

To Dr. M. Wanyoike, Dr. C. Kariuki, Dr. D. Silverstein, Dr. M. Warshow, Dr. R. Mathenge, and their respective clinics and patients. Sr Ngaruiya of Nairobi Hospital Catheterization Laboratory; E. Mulama of the Nairobi Hospital and E. Alonya of Nairobi Heart Clinic.

\section{REFERENCES}

1. Murray, C. J. L. and Lopez, A. D. The Global Pattern of Disease, In: Murray C.J.L, Lopez A.D. (Eds): The Global Burden of Disease: A Comprehensive Assessment of Mortality and Disability From Disease, Injuries and Risk factors in 1990 and projected to 2020. USA, Harvard School of Health, 1996; pp. 1-52.

2. Woolf, N. Pathology of atherosclerosis. London, 1982; Butterworths, pp 150, 157

3. Gillum, R. F. and Grant, C. T. Coronary heart disease in black populations. II. Risk factors. Amer. Heart. J. 1982; 104: 852.

4. The sixth report of the Joint National Committee on Detection, Evaluation, and Treatment of High Blood Pressure, JNC VI: Arch. Intern. Med. 1997; 157: 2413-2446.
5. Summary of the Second Report of the National Cholesterol Education Programme (NCEP) Expert Panel on Detection, Evaluation, and Treatment of High Blood Cholesterol In Adults (Adult Treatment panel II). JAMA, 1993; 269: 3015.

6. J.J., Pompa, Bittl, J. Coronary Angiography and Intravascular Ultrasonography. In: Braunwald (Ed) Heart Diseases. 6th Ed 2001.

7. Gikonyo, D., Wanyoike, M., Gikonyo, B. and Wariua, G. The Cardiac Catheterization Laboratory, The Nairobi Hospital. The Nairobi Hospital Proceedings. Vol. 5: 186.

8. Reaven, G. M. Banting Lecture 1988. Role of insulin resistance in human disease. Diabetes 1988; 37:1595.

9. Zavaroni, I., Bonora, E., Pagliara, M., et al. Risk factors for coronary artery disease in healthy persons with hyperinsulinemia and normal glucose tolerance. N. Engl. J. Med. 1989; 320:702.

10. Kannel, W. B.and McGee D. L. Diabetes and cardiovascular risk factors: The Framingham Study. Circulation, 1979; 59:8

11. Singer, D. E., Nathan, D. M., Anderson, K. M., et al. Association of $\mathrm{HbA} 1 \mathrm{c}$ with prevalent cardiovascular disease in the original cohort of the Framingham Heart Study. Diabetes 1992; 41:202.

12. I8th Bethesda Conference Report: Cardiovascular disease in the elderly. May 8-10, 1986. J. Am. Coll. Cardiol. 1987; 10:1A.

13. The sixth report of the Joint National Committee on Detection, Evaluation, and Treatment of High Blood Pressure, JNC VI: Arch. Intern. Med. 997; 157:2413-2446.

14. Yonga, G., Ogola, E. and Juma, F. Cardiovascular risk factor profiles in mild to moderate hypertensives seen at Kenyatta National Hospital. East Afr. Med. J. 1993; 70:693-695.

15. Collins, R., Peto, R., MacMahon, S., et al. Blood pressure, stroke and coronary heart disease. Part 2. Short term reductions in blood pressure. Lancet, 1990; 335.

16. Vaghela, V. P. Cardiovascular risk factors associated with type 2 Diabetes Mellitus as seen at the Kenyatta National Hospital. -Dissertation for degree of MMed (Int. Med) 2001. University of Nairobi.

17. Kadiri, S. and Salako, B. L. Cardiovascular risk factors in middle aged Nigerians. East Afr. Med. J. 1997; 74: 303-306. 